\title{
Statistical Analysis of Fragility Curves
}

\section{By Masanobu Shinozuka, ${ }^{1}$ Honorary Member, M. Q. Feng, ${ }^{2}$ Associate Member, ASCE, Jongheon Lee, ${ }^{3}$ and Toshihiko Naganuma ${ }^{4}$}

\begin{abstract}
AвStRACt: This paper presents a statistical analysis of structural fragility curves. Both empirical and analytical fragility curves are considered. The empirical fragility curves are developed utilizing bridge damage data obtained from the 1995 Hyogo-ken Nanbu (Kobe) earthquake. The analytical fragility curves are constructed on the basis of the nonlinear dynamic analysis. Two-parameter lognormal distribution functions are used to represent the fragility curves with the parameters estimated by the maximum likelihood method. This paper also presents methods of testing the goodness of fit of the fragility curves and estimating the confidence intervals of the two parameters (median and log-standard deviation) of the distribution. An analytical interpretation of randomness and uncertainty associated with the median is provided.
\end{abstract}

\section{INTRODUCTION}

In performing a seismic risk analysis of a structural system, it is imperative to identify seismic vulnerability of component structures associated with various states of damage. The development of vulnerability information in the form of fragility curves is a widely practiced approach when the information is to be developed accounting for a multitude of uncertain sources involved, for example, in estimation of seismic hazard, structural characteristics, soil-structure interaction, and site conditions. In principle, the development of fragility curves will require synergistic use of the following methods: (1) Professional judgment; (2) quasi-static and design code consistent analysis; (3) utilization of damage data associated with past earthquakes; and (4) numerical simulation of the seismic response of structures based on dynamic analysis.

This paper concentrates on the development of empirical and analytical fragility curves for bridges as described in Methods 3 and 4 above, respectively - the former by utilizing the damage data associated with a past earthquake, and the latter by numerically simulating seismic response with the aid of structural dynamic analysis. At the same time, this paper introduces statistical procedures appropriate for the development of fragility curves under the assumption that they can be represented by two-parameter lognormal distribution functions. These procedures describe how the test of goodness of fit can be performed and the confidence intervals of the two parameters can be estimated. The empirical fragility curves are developed utilizing bridge damage data obtained from the 1995 Hyogo-ken Nanbu (Kobe) earthquake. Analytical fragility curves are developed for typical bridges in the Memphis area on the basis of a nonlinear dynamic analysis.

Two-parameter lognormal distribution functions were traditionally used for fragility curve construction. This was motivated by its mathematical convenience in relating the actual structural strength capacity with the design strength primarily through a seismic factor of safety, which can be factored into a number of multiplicative safety factors, each associated with

${ }^{1}$ Fred Champion Prof., Dept. of Civ. Engrg., Univ. of Southern California, University Park, CA 90089-2531.

${ }^{2}$ Assoc. Prof., Dept. of Civ. and Envir. Engrg., Univ. of California, Irvine, CA 92697-2175.

${ }^{3}$ Visiting Prof., Dept. of Civ. and Envir. Engrg., Univ. of California, Irvine, CA.

${ }^{4}$ Mgr. of Survey and Des. Div., Hanshin Expressway Public Corp., Osaka, Japan

Note. Associate Editor: Ahsan Kareem. Discussion open until May 1, 2001. To extend the closing date one month, a written request must be filed with the ASCE Manager of Journals. The manuscript for this paper was submitted for review and possible publication on July 21, 1999. This paper is part of the Journal of Engineering Mechanics, Vol. 126, No. 12, December, 2000. (C)ASCE, ISSN 0733-9399/00/0012-1224-1231/ $\$ 8.00+\$ .50$ per page. Paper No. 21465 . a specific source of randomness and/or uncertainty. When the lognormal assumption is made for each of these factors, the overall seismic safety factor also distributes lognormally due to the multiplicative reproducibility of the lognormal variables. This indeed was the underpinning assumption that was made in the development of probabilistic risk assessment methodology for nuclear power plants in the 1970s and in the early 1980s [U.S. Nuclear Regulatory Commission (U.S. NRC) 1983].

\section{EMPIRICAL FRAGILITY CURVES}

Empirical fragility curves for the Hanshin Expressway Public Corporation's (HEPC's) bridges (columns) are developed on the basis of the records of the damage resulting from the 1995 Kobe earthquake. It is assumed that the curves can be expressed in the form of two-parameter lognormal distribution functions, and the estimation of the two parameters (median and log-standard deviation) is performed with the aid of the maximum likelihood method. For this purpose, the peak ground acceleration (PGA) is used to represent the intensity of the seismic ground motion, although use of intensity measures other than PGA such as peak ground velocity, spectral acceleration, spectral intensity, and modified Mercalli intensity are possible.

The likelihood function for the present purpose is expressed as follows:

$$
L=\prod_{i=1}^{N}\left[F\left(a_{i}\right)\right]^{x_{i}}\left[1-F\left(a_{i}\right)\right]^{1-x_{i}}
$$

where $F(\cdot)$ represents the fragility curve for a specific state of damage; $a_{i}=$ PGA value to which bridge $i$ is subjected; $x_{i}=$ 1 or 0 depending on whether or not the bridge sustains the state of damage under PGA $=a_{i}$; and $N=$ total number of bridges inspected after the earthquake. Under the current lognormal assumption, $F(a)$ takes the following analytical form:

$$
F(a)=\Phi\left[\frac{\ln \left(\frac{a}{c}\right)}{\zeta}\right]
$$

in which $a$ represents PGA; and $\Phi[\cdot]=$ standardized normal distribution function.

The two parameters $c$ and $\zeta$ in (2) are computed as $c_{e}$ and $\zeta_{e}$ satisfying the following equations to maximize $\ln L$ and hence $L$ :

$$
\frac{d \ln L}{d c}=\frac{d \ln L}{d \zeta}=0
$$

This computation is performed by implementing a straightforward optimization algorithm. 
Fragility curves are constructed (Nakamura et al. 1998) on the basis of a sample of 770 single-support reinforced concrete columns along two stretches of the viaduct, one in the HEPC's Kobe Route and the other in the Ikeda Route with a total length of $40 \mathrm{~km}$. The damage data reported by HEPC's engineers after the 1995 Kobe earthquake are utilized for this purpose. The state of damage is classified as collapse $\left(A_{s}\right)$, major $(A)$, moderate $(B)$, and minor $(C)$. These bridge columns

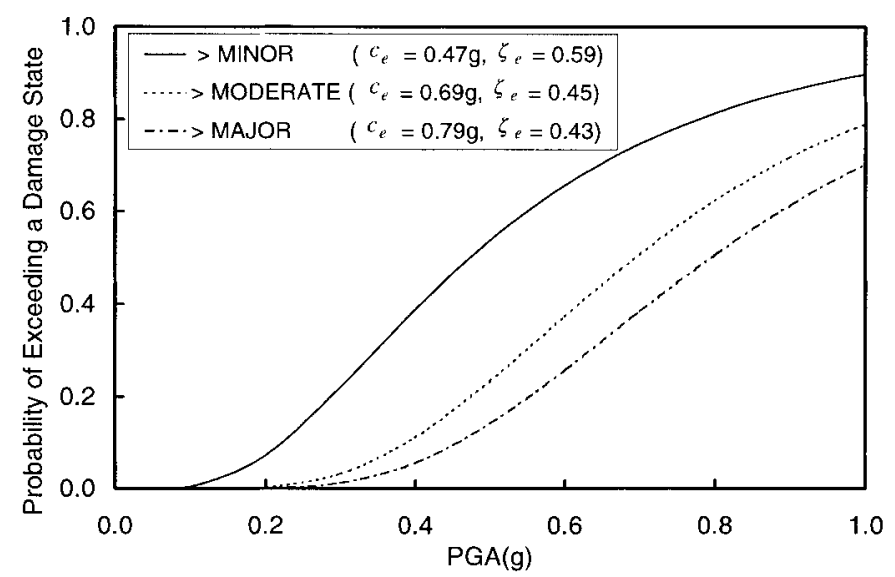

FIG. 1. Fragility Curves for HEPC's Bridges are of similar geometry and are similarly reinforced. In this respect, the 770 columns under consideration here constitute approximately a homogeneous statistical sample. The PGA value at each column location under the Kobe earthquake is estimated by Nakamura et al. (1998) on the basis of the work by Nakamura and Mizatani (1996).

Integrating the damage state information with that of the PGA, and making use of the maximum likelihood method involving (1)-(3), three fragility curves are constructed as shown in Fig. 1 together with values of the median $c_{e}$ and $\log$ standard deviation $\zeta_{e}$. The curve with a "minor" designation represents, at each PGA value $a$, the probability that "at least a minor" state of damage will be sustained by a bridge (arbitrarily chosen from the sample of bridges) when it is subjected to PGA $a$. The same meaning applies to other curves with their respective damage state designations.

\section{ANALYTICAL FRAGILITY CURVES}

To demonstrate the development of analytical fragility curves, two representative bridges with a precast, prestressed continuous deck in the Memphis area studied by Jernigan and Hwang (1997) are used. The plan, elevation, and column cross section of Bridge 1 are depicted in Fig. 2. Geometry and configuration of Bridge 2 are similar to Bridge 1. Bridge 2 also has a precast, prestressed continuous deck. However, the deck

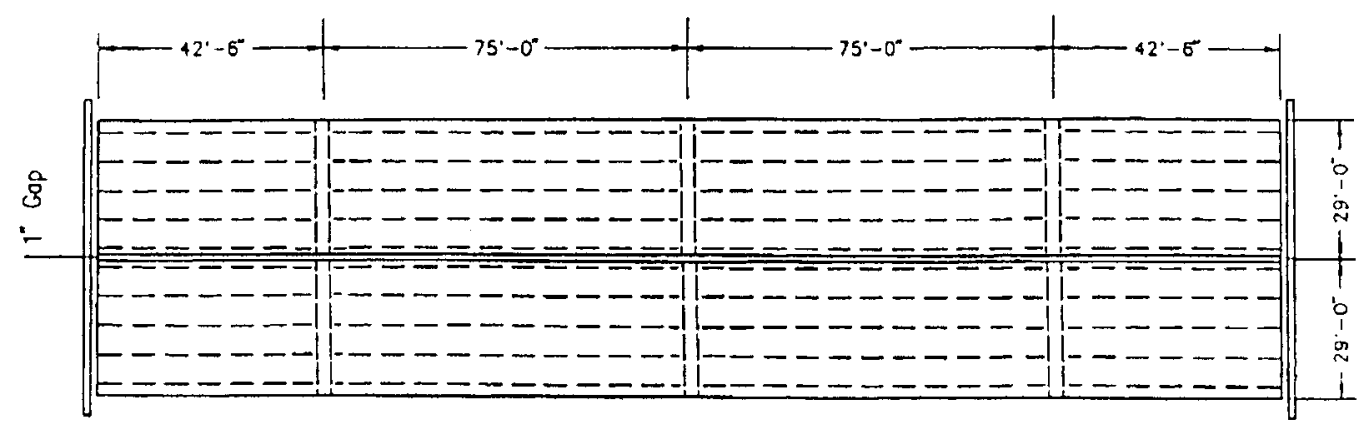

$\underline{\text { Plan }}$

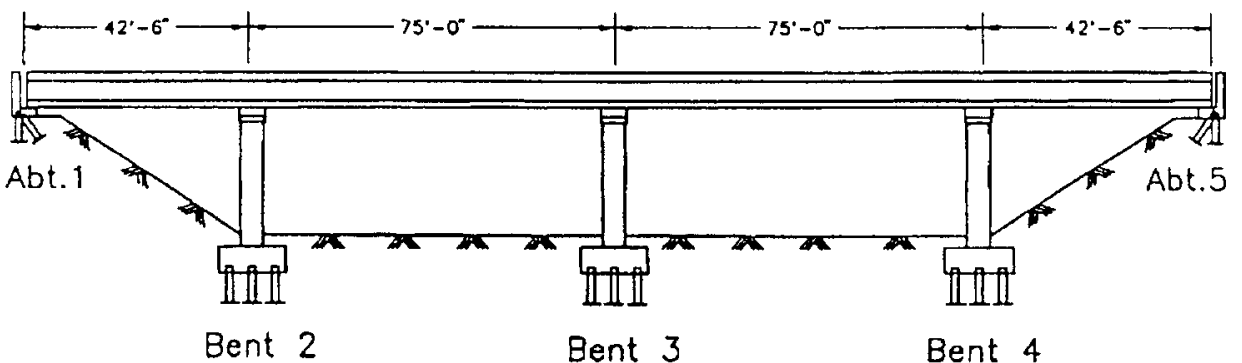

Elevation

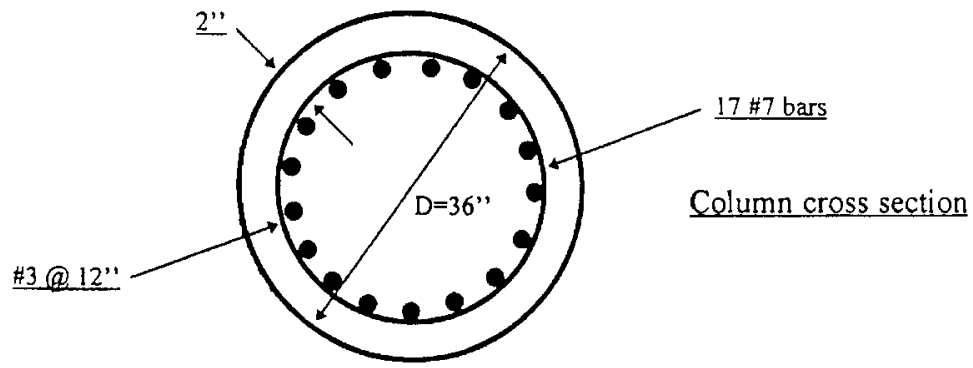

FIG. 2. Representative Memphis Bridge (Bridge 1) 

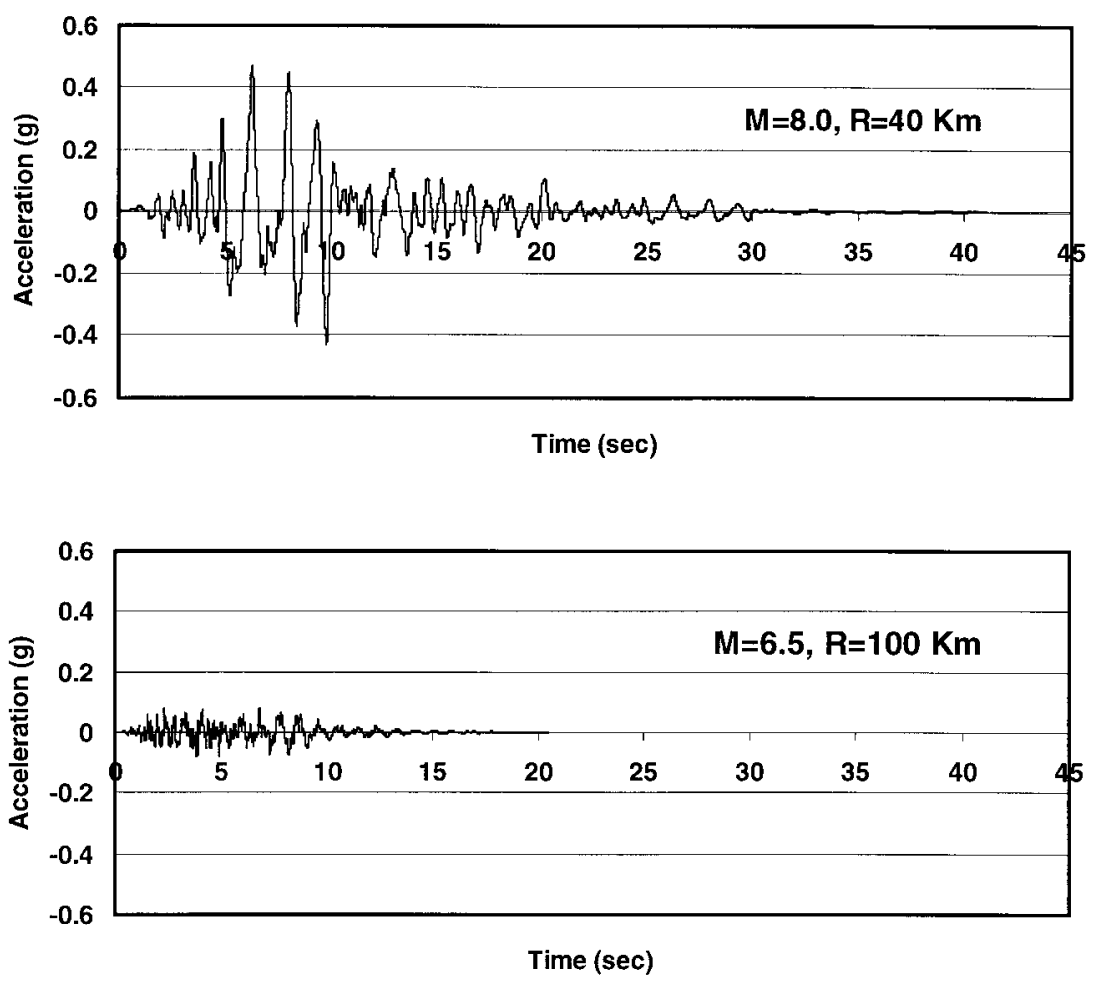

FIG. 3. Typical Ground Motion Time Histories

is supported by two abutments and four bents with five spans equal to $10.7 \mathrm{~m}(35 \mathrm{ft}), 16.8 \mathrm{~m}(55 \mathrm{ft}), 16.8 \mathrm{~m}(55 \mathrm{ft}), 16.8$

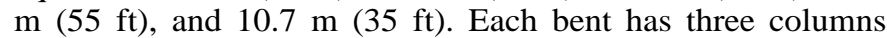
$5.8 \mathrm{~m}(19 \mathrm{ft})$ high with the same cross-sectional and reinforcing characteristics as those of Bridge 1. Following Jernigan and Hwang (1997), the strength $f_{c}$ of $20.7 \mathrm{MPa}(3,000 \mathrm{psi})$ concrete used for the bridge is assumed to be best described by a normal distribution with a mean strength of $31.0 \mathrm{MPa}$ (4,500 psi) and a standard deviation of $6.2 \mathrm{MPa}$ (900 psi), whereas the yield strength $f_{y}$ of grade 40 reinforcing bars used in the design is described by a lognormal distribution having a mean strength of $336.2 \mathrm{MPa}(48.8 \mathrm{ksi})$ with a standard deviation of $36.0 \mathrm{MPa}(5.22 \mathrm{ksi})$. Then, a sample of 10 "nominally identical but statistically different" bridges are created for each of Bridges 1 and 2 by simulating 10 realizations of $f_{c}$ and $f_{y}$ according to the respective probability distribution functions assumed. Other parameters that could contribute to variability of structural response were not considered in the present analysis under the assumption that their contributions can be disregarded.

For the seismic ground motion, the time histories generated by Hwang and Huo (1996) at the Center for Earthquake Research and Information at the University of Memphis are used. These time histories are generated by making use of the Fourier acceleration amplitude on the base rock derived under the assumption of a far-field point source by Boore (1983). In fact, the study area is located $40-100 \mathrm{~km}$ from Marked Tree, Ark., the epicenter of an 1846 earthquake of magnitude 6.5 and of all the scenario earthquakes considered in this study. Upon using seismologically consistent values for the parameters in the Boore model and other related models and converting the Fourier amplitude to the power spectrum, corresponding histories are generated in terms of sample functions of a normal (Gaussian) stationary process on the base rock by means of the spectral representation method by Shinozuka and Deodatis (1991). The seismic wave represented by these time histories is propagated through the surface layer to the ground surface by means of the SHAKE 91 computer code by Idriss and Sun (1992) and used upon appropriately modulating in the time

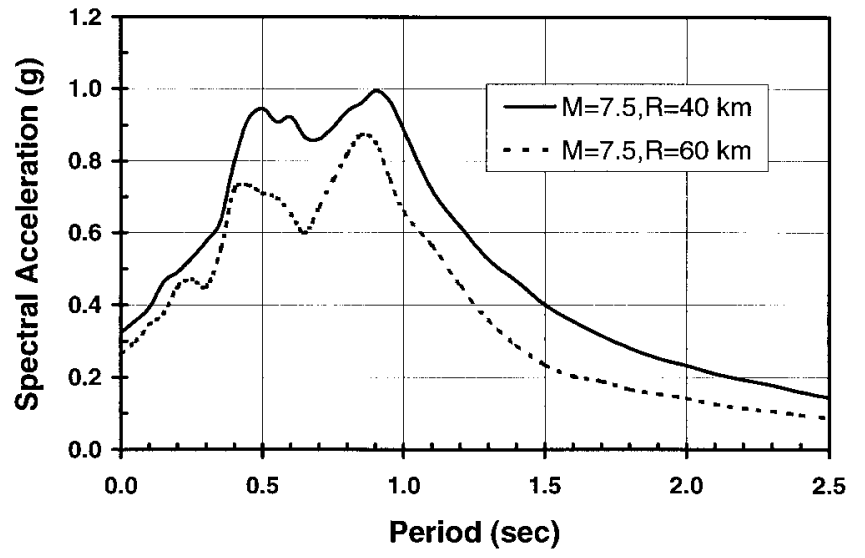

FIG. 4. Average Acceleration Response Spectra

domain (Hwang and Huo 1996) for the response analysis. To minimize computational effort, samples of 10 time histories are randomly selected from 50 histories generated by Hwang and Huo (1996) for each of the following eight combinations of magnitude $(M)$ and epicentral distance $(R): M=6.5$ with $R=80$ and $100 \mathrm{~km}, M=7.0$ with $R=60$ and $80 \mathrm{~km}, M=$ 7.5 with $R=40$ and $60 \mathrm{~km}$, and $M=8.0$ with $R=40$ and 60 $\mathrm{km}$.

Typical ground motion time histories for two extreme combinations $M=8.0$ with $R=40 \mathrm{~km}$ and $M=6.5$ with $R=100$ $\mathrm{km}$ are shown in Fig. 3. The spectral accelerations averaged over 10 acceleration time histories used in this study from each of the combinations of $M=7.5$ for $R=40$ and $60 \mathrm{~km}$ is shown in Fig. 4 to provide insight to the frequency content of these ground motion time histories. For the purpose of response analysis, a sample of 10 time histories generated from each $M$ and $R$ combination is matched with a sample of 10 bridges in a pseudo-Latin hypercube format. Hence, each statistical representation of Bridges 1 and 2 is subjected to 80 ground motion time histories.

The present study utilizes the SAP 2000 finite-element code, 


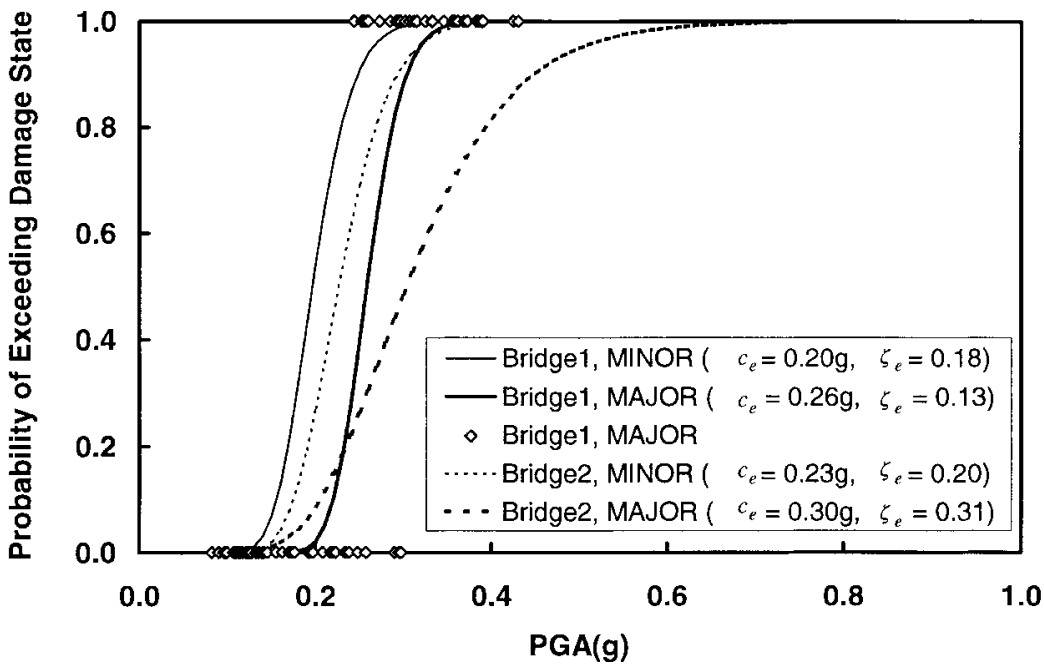

FIG. 5. Fragility Curves for Memphis Bridges

which can in approximation simulate the state of damage of each bridge under a ground acceleration time history. This computer code can provide hysteretic elements that are in essence bilinear without strength or stiffness degeneration.

The states of damage considered for Bridges 1 and 2 are major (all of the columns subjected to ductility demand $\geq 2$ ) and "at least minor" (all of the columns subjected to ductility demand $\geq 1$ ) under the longitudinal applications of ground motion. Fig. 5 shows the fragility curves associated with these states of damage for Bridges 1 and 2. Eighty diamonds plotted on the two horizontal axes represent $x_{i}=0$ (for the state of no damage) and $x_{i}=1$ (for the state of major damage) in relation to (1) for Bridge 1 under the 80 earthquakes generated. The corresponding fragility curves are derived on the basis of these diamonds in conjunction with (1)-(3). The analysis performed under the ground motion in the transverse direction produced the states of lesser damage and hence are not reported in this paper.

\section{STATISTICAL ANALYSIS}

To date, the issues of hypothesis testing and confidence intervals relating to fragility curve development have not been addressed in the literature, primarily because the earthquake engineering community has never had the opportunity to collect damage data of a sufficiently large sample that can be used to develop fragility curves on the basis of legitimate statistical analysis. However, the 1994 Northridge and 1995 Kobe earthquakes, inflicting devastating damage upon many bridges, buildings, port facilities, and other engineered structures, made it possible to consider statistical methods to analyze the probabilistic characteristics of damage in a more judicious fashion rather than relying on an ad hoc curve-fitting exercise.

\section{Test of Goodness of Fit}

The fundamental probabilistic interpretation of a fragility curve $F(a)$ as a function of $a$ suggests that a bridge will sustain a designated state of damage with probability $F(a)$ and will not sustain the damage state with probability $1-F(a)$ under the earthquake intensity represented by PGA equal to $a$. This means that, under each PGA value, the probabilistic phenomena one deals with can be described by random variable $X_{i}$ following the Bernoulli distribution such that $X_{i}=1$ when the state of damage is reached under PGA $=a_{i}$, and $X_{i}=0$ otherwise. Then

$$
Y_{i}^{2}=\left(X_{i}-p_{i}\right)^{2}
$$

has mean and variance equal to

$$
\begin{aligned}
\mu_{Y_{i}^{2}} & =p_{i}\left(1-p_{i}\right) \\
\sigma_{Y_{i}^{2}}^{2}=\operatorname{var}\left(Y_{i}^{2}\right) & =p_{i}\left(1-p_{i}\right)\left(1-2 p_{i}\right)^{2}
\end{aligned}
$$

respectively, where $p_{i}=F\left(a_{i}\right)$.

The sum of $Y_{i}^{2}$ shown below

$$
Y^{2}=\sum_{i=1}^{N}\left(X_{i}-p_{i}\right)^{2}
$$

approaches asymptotically normal (Gaussian) as $N$ becomes large due to the central limit theorem as each Bernoulli event under consideration is assumed independent, where $N$ is the sample size (the total number of the bridges considered), and in this analysis it is indeed a large value $(>>1)$.

Recalling that $X_{i}$ is independent of $X_{j}(i \neq j)$ and governed by the Bernoulli distribution, a straightforward analysis shows that the expected value $\mu_{Y^{2}}=\mathrm{E}\left(Y^{2}\right)$ and the variance $\sigma_{Y^{2}}^{2}=$ $\operatorname{var}\left(Y^{2}\right)$ can be written as follows:

$$
\begin{gathered}
\mu_{Y^{2}}=\mathrm{E}\left(Y^{2}\right)=\sum_{i=1}^{N} p_{i}\left(1-p_{i}\right) \\
\sigma_{Y^{2}}^{2}=\operatorname{var}\left(Y^{2}\right)=\sum_{i=1}^{N} p_{i}\left(1-p_{i}\right)\left(1-2 p_{i}\right)^{2}
\end{gathered}
$$

On the other hand, if $x_{i}$ represents the realization observation of $X_{i}$ as defined in the likelihood function given by (1)

$$
y^{2}=\sum_{i=1}^{N}\left(x_{i}-p_{i}\right)^{2}
$$

is the realization of $Y^{2}$.

Because $p_{i}$ depends on the values of $c_{e}$ and $\zeta_{e}$, the standard procedure of hypothesis testing suggests that if $\alpha$ is a significance level and

$$
P_{y^{2}}=\Phi\left(\frac{y^{2}-\mu_{y^{2}}}{\sigma_{y^{2}}}\right) \leq 1-\alpha
$$

TABLE 1. $P_{y^{2}}$ Values for Goodness of Fit

\begin{tabular}{l|c|c|c}
\hline \hline $\begin{array}{c}\text { Damage } \\
\text { state } \\
(1)\end{array}$ & $\begin{array}{c}\text { HEPC's } \\
\text { bridges } \\
(2)\end{array}$ & $\begin{array}{c}\text { Memphis } \\
\text { Bridge 1 } \\
(3)\end{array}$ & $\begin{array}{c}\text { Memphis } \\
\text { Bridge 2 } \\
(4)\end{array}$ \\
\hline Minor & 0.39 & 0.64 & 0.48 \\
Moderate & 0.86 & - & - \\
Major & 0.73 & 0.57 & 0.64 \\
\hline \hline
\end{tabular}




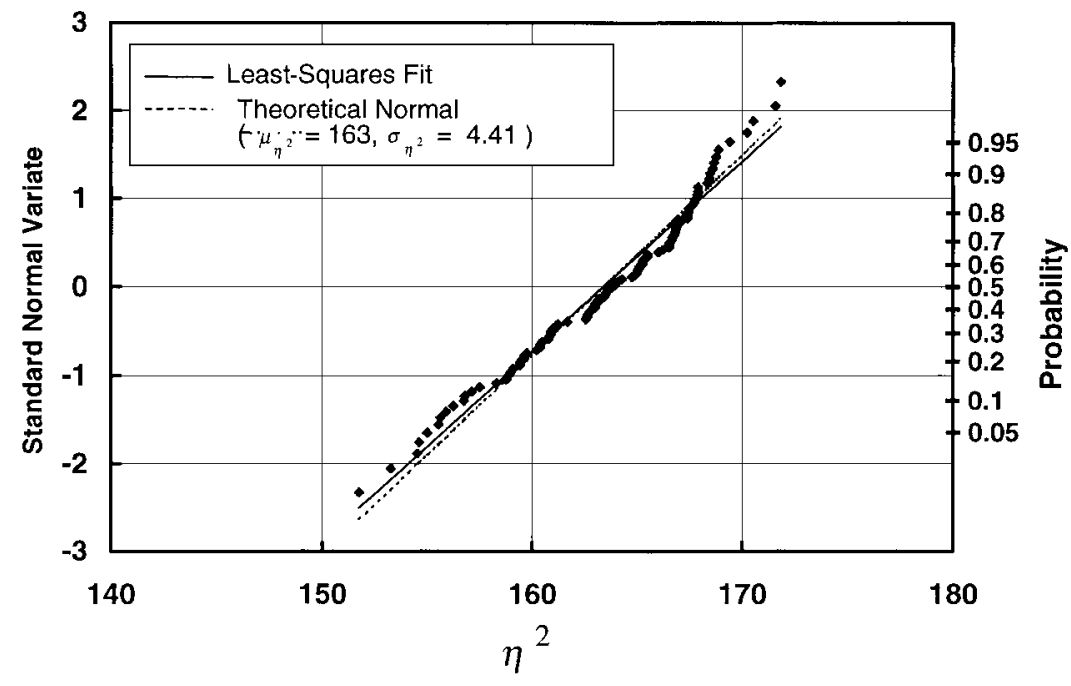

FIG. 6. Validation of $\eta^{2}$ Asymptotically Approaching Normal Distribution

then, the hypothesis that $c_{e}$ and $\zeta_{e}$ are indeed the true values of $c$ and $\zeta$ cannot be rejected at the significance level $\alpha$ usually set equal to 0.05 or 0.10 .

The $p_{y^{2}}$ values for the fragility curve developed for HEPC's bridges (Fig. 1), and Memphis Bridges 1 and 2 (Fig. 5) are given in Table 1 . These values indicate that the hypotheses involved in all of the cases cannot be rejected at the significance level of $10 \%$. It is noted here that this method can test the goodness of fit of the fragility curve only over the range of PGA where damage data sufficiently exist. For example, the fragility curve for Bridge 1 associated with the state of major damage in Fig. 5 is not necessarily valid beyond $0.4 g$. A simplistic parallel to this is the case of extrapolating the result of least-squares curve fitting much beyond the range of existing data.

Fig. 6 shows the validity of the assumption of $Y^{2}$ in (7) being asymptotically normal by means of plotting the 100 realizations of $Y^{2}$. This requires simulation of $X_{i}$ at each $a_{i}$ using $p_{i}$ based on $c_{e}$ and $\zeta_{e}$ obtained from the empirically or analytically observed damage data. Upon simulating all $X_{i}$ for all $a_{i}$ and obtaining their realizations $\xi_{i},(10)$ is evaluated as

$$
\eta^{2}=\sum_{i=1}^{N}\left(\xi_{i}-p_{i}\right)^{2}
$$

where $\eta$ and $\xi_{i}$ are written in place of $y$ and $x_{i}$ to distinguish the simulated data from the actual data. This process is repeated $k$ times ( $k=100$ here) to produce 100 realizations of $Y^{2}$ consisting of the same number of $\eta^{2}$, each representing one set of simulations of $\xi_{i}(i=1,2, \ldots, N)$. This sample of $\eta^{2}$ is indeed plotted in Fig. 6 using the normal probability paper. The dashed line represents the least-squares fit of the sample, and the solid line indicates the theoretical normal distribution with the mean and standard deviation given by (8) and (9), respectively, for the fragility curve for HEPC's bridges associated with the state of "at least minor" damage. For other fragility curves demonstrated above, the simulated realizations of $Y^{2}$ can also be shown to distribute in accordance with normal distributions with their respective mean and standard deviation derivable from (8) and (9).

\section{Estimation of Confidence Intervals}

The estimators $\hat{c}$ and $\hat{\zeta}$ of $c$ and $\zeta$ cannot be explicitly given in terms of analytical forms as they represent optimal solutions obtained numerically by solving (3). From the uncertainty analysis point of view, however, it is most desirable to demonstrate the extent of the statistical variations of these esti- mators. To this end, Monte Carlo simulation techniques are used to generate realizations of $\hat{c}$ and $\hat{\zeta}$. These call for the same simulation of $X_{i}$ at each $a_{i}$ using $p_{i}$ based on $c_{e}$ and $\zeta_{e}$ as exercised to demonstrate the asymptotic normal property of $Y^{2}$ in relation to the test of goodness of fit. Upon simulating $X_{i}$ for all $a_{i}$ and obtaining their realizations $\xi_{i}(i=1,2, \ldots$, $N)$, (3) is solved for $c_{e}$ and $\zeta_{e}$ as a set of realizations of $\hat{c}$ and $\hat{\zeta}$.

Repeating this process a large number of times (500 times in this case), one obtains 500 sets of realizations of $\hat{c}$ and $\hat{\zeta}$ as plotted in Fig. 7. Assuming that the marginal distribution of $\hat{c}$ is lognormal, and taking the $90 \%$ confidence interval between $\hat{c}=c_{0.95}$ and $\hat{c}=c_{0.05}$ associated with exceedance probabilities 95 and $5 \%$ of $\hat{c}$, one obtains $c_{0.95}=0.45 g$ and $c_{0.05}=$ $0.50 \mathrm{~g}$ for the fragility curve of HEPC's bridges with the state of "at least minor" damage. Fig. 8 shows the three fragility curves with medians $c=c_{0.95}, c=c_{e}$, and $c_{0.05}$ with the identical log-standard deviation $\zeta_{0}=0.59$; the curves on the left and right, respectively, represent the fragility curves with 95 and $5 \%$ confidence. Following the tradition of the risk assessment procedure for the nuclear power plant (U.S. NCR 1983), the $\log$-standard deviations $\zeta=\zeta_{e}$ associated with $c=c_{e}$ is used for these three curves, although it is possible to combine $\zeta=$ $\zeta_{0.95}$ and $\zeta=\zeta_{0.05}$, which are derivable from the simulated distribution of $\hat{\zeta}$, together with $c=c_{0.95}$ and $c=c_{0.05}$, respectively. This study contends as in "PRA Procedures Guide" (U.S. NRC 1983) that this is justifiable because the variation in $c$ has the first-order effect on fragility values whereas that in $\zeta$ has the second-order effect in general. Similar fragility curves with 95 and 5\% confidence are obtained for other states of damage.

In the probabilistic risk assessment of nuclear power plants,

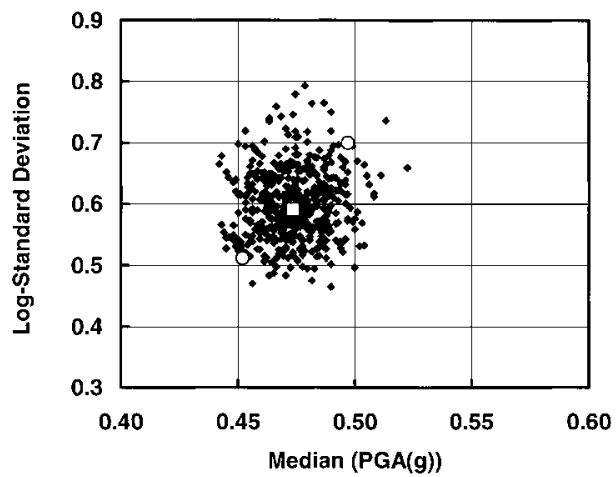

FIG. 7. Simulated Distribution of $\hat{c}$ and $\hat{\zeta}$ 


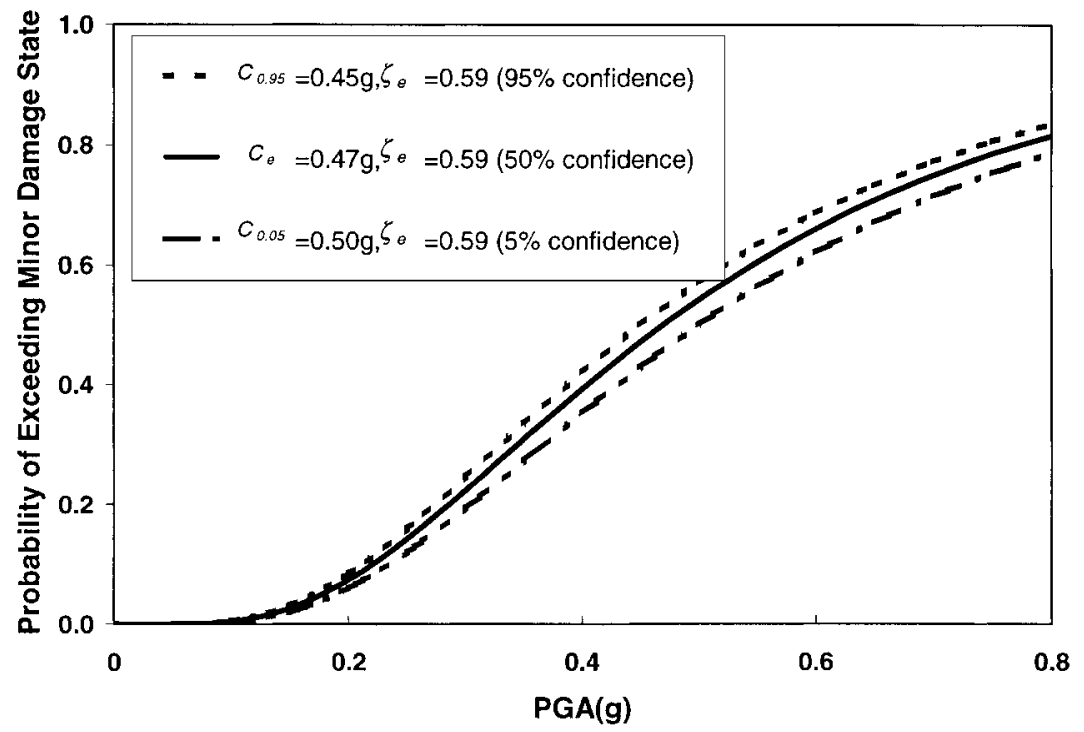

FIG. 8. Confidence Bands of 95 and $5 \%$ for HEPC's Fragility Curve

an "average" fragility curve $F^{*}(a)$ is derived and utilized often. This average curve is obtained by unconditionalizing $F(a \mid c)=\Phi[\ln (a / c) / \zeta]$ under the assumption that $c$ is lognormally distributed

$$
F^{*}(a)=\int_{z} F(a \mid z) f_{c}(z) d z
$$

where $f_{c}(z)=$ lognormal density function of $c$ with median $\tilde{c}$ and log-standard deviation $\zeta_{c}$. The distribution function $F^{*}(a)$ is not lognormal. However, in approximation, it may be and, indeed, was used in practice as lognormal distribution in "PRA Procedures Guide" (U.S. NRC 1983)

$$
f_{c}(z)=\Phi\left[\frac{\ln (z / \tilde{c})}{\zeta_{c}}\right]
$$

The distribution function $F^{*}(a)$ has the same statistical significance as the combined or composite fragility curve to be introduced below.

\section{Development of Combined and Composite Fragility Curves}

Use of a fragility curve representing a family of bridges with similar structural attributes, primarily categorized in specific structural types, expedites the process of urban earthquake disaster estimation. A well-known example of such a categorization is found in ATC 13 [Applied Technology Council (ATC) 1985]. Bridges 1 and 2 in the Memphis area analyzed in an earlier section belong to such a family of bridges that can be categorized as precast, prestressed continuous deck bridges with short to medium length. This section demonstrates how combined fragility curves for a category of bridges can be derived from individual fragility curves constructed for member bridges in the aforementioned category.

The fragility curves (associated with specific states of damage) analytically developed for Bridges 1 and 2 in the Memphis area can be combined in the following fashion in order to develop a combined fragility curve for a mixed set of population of bridges in which there are $N_{1}$ and $N_{2}$ of Bridges 1 and 2 , respectively. In this case, the combined fragility curve $F_{c}(a)$ is obtained as

$$
F_{c}(a)=P_{1} \cdot F_{1}(a)+P_{2} \cdot F_{2}(a)
$$

where $F_{i}(a)=$ fragility curve for Bridge $i$ and

$$
P_{i}=\frac{N_{i}}{\left(N_{1}+N_{2}\right)}
$$

is the probability with which a Bridge $i$ will be chosen at random from the combined population. With $N_{1}=N_{2}=1$, the resulting fragility curve $F_{c}(a)$ for major damage is shown in Fig. 9. It is noted that $F_{c}(a)$ thus developed is no longer a lognormal distribution.

Because the density function $f_{c}(a)$ of $F_{c}(a)$ can be written

$$
f_{c}(a)=P_{1} f_{1}(a)+P_{2} f_{2}(a)
$$

and because $f_{i}(a)$ is a lognormal density function, the expected value of $\alpha=\ln a$ for the combined distribution is given by

$$
\bar{\alpha}=P_{1} \ln c_{1}+P_{2} \ln c_{2}=P_{1} \bar{\alpha}_{1}+P_{2} \bar{\alpha}_{2}
$$

in which $c_{i}=$ median associated with $f_{i}(a)$ and

$$
\bar{\alpha}_{i}=\ln c_{i}
$$

If $\bar{\alpha}$ is defined as

$$
\bar{\alpha}=\ln a_{c}
$$

then

$$
a_{c}=c_{1}^{P_{1}} \cdot c_{2}^{P_{2}}
$$

To obtain the standard deviation $\zeta_{c}$ of $\alpha=\ln a$ of the combined distribution, one recognizes

$$
\begin{gathered}
\zeta_{c}^{2}=\mathrm{E}(\alpha-\bar{\alpha})^{2}=\mathrm{E}\left(\alpha^{2}\right)-\bar{\alpha}^{2} \\
\mathrm{E}\left(\alpha^{2}\right)=P_{1} \int \alpha^{2} \varphi_{1}(\alpha) d \alpha+P_{2} \int \alpha^{2} \varphi_{2}(\alpha) d \alpha \\
=P_{1} \mathrm{E}_{1}\left(\alpha^{2}\right)+P_{2} \mathrm{E}_{2}\left(\alpha^{2}\right)
\end{gathered}
$$

where $\varphi_{i}(\alpha)=$ normal density function of $\alpha$ with mean $\ln c_{i}$ and standard deviation $\zeta_{i}$. One further recognizes that

$$
\mathrm{E}_{i}(\alpha-\bar{\alpha})^{2}=\int(\alpha-\bar{\alpha})^{2} \varphi_{i}(\alpha) d \alpha=\mathrm{E}_{i}\left(\alpha^{2}\right)-\bar{\alpha}_{i}^{2}=\zeta_{i}^{2}
$$

from which it follows that 


$$
\mathrm{E}_{i}\left(\alpha^{2}\right)=\int \alpha^{2} \varphi_{i}(\alpha) d \alpha=\zeta_{i}^{2}+\bar{\alpha}_{i}^{2}
$$

Combining (18) and (22)-(25)

$$
\begin{aligned}
\zeta_{c}^{2} & =P_{1} \zeta_{1}^{2}+P_{2} \zeta_{2}^{2}+P_{1}\left(1-P_{1}\right) \bar{\alpha}_{1}^{2}+P_{2}\left(1-P_{2}\right) \bar{\alpha}_{2}^{2} \\
& -2 P_{1} P_{2} \bar{\alpha}_{1} \bar{\alpha}_{2}
\end{aligned}
$$

The last three terms of the right-hand side of (26) are positive semidefinite with respect to $\bar{\alpha}_{1}$ and $\bar{\alpha}_{2}$. In fact, the sum of these terms are positive except when $\bar{\alpha}_{1}=\bar{\alpha}_{2}$, in which case the sum is equal to zero. This indicates that the combination of two fragility curves produces a variance that includes the terms that form a quadratic expression of logarithms of the medians, which always increases the value of the variance from its minimum equal to the sum of the first two terms of the right-hand side of (26), unless the medians are equal.

Eqs. (15)-(18) and (26) can all be easily generalized and take the following forms:

$$
\begin{gathered}
F_{c}(a)=\sum_{i=1}^{M} P_{i} F_{i}(a) \\
P_{i}=\frac{N_{i}}{N}
\end{gathered}
$$

where $N=\sum_{i=1}^{M} N_{i}=$ total number of bridges with $N_{i}$ being the number of Bridge $i$ or the $i$ th nominally identical but statistically different set of bridges in the population of a category of bridges

$$
\begin{gathered}
f_{c}(a)=\sum_{i=1}^{M} P_{i} f_{i}(a) \\
\bar{\alpha}=\ln a_{c}=\sum_{i=1}^{M} P_{i} \bar{\alpha}_{i} \\
a_{c}=\prod_{i=1}^{M} c_{i}^{P_{i}} \\
\zeta_{c}^{2}=\mathbf{P}^{T} \mathbf{Z}+\mathbf{A}^{T} \mathbf{Q A}
\end{gathered}
$$

where

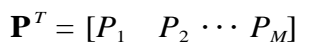

$$
\begin{gathered}
\mathbf{Z}^{T}=\left\lfloor\begin{array}{llll}
\zeta_{1}^{2} & \zeta_{2}^{2} & \cdots & \zeta_{M}^{2}
\end{array}\right] \\
\mathbf{Q}=\left[\begin{array}{cccc}
\mathbf{A}^{T}=\left[\begin{array}{cccc}
\alpha_{1} & \alpha_{2} & \cdots & \alpha_{M}
\end{array}\right] \\
P_{1}\left(1-P_{1}\right) & -P_{1} P_{2} & \cdots & -P_{1} P_{M} \\
\vdots & & & \\
\vdots & & & \\
-P_{m} P_{1} & -P_{M} P_{2} & \cdots & P_{M}\left(1-P_{M}\right)
\end{array}\right]
\end{gathered}
$$

The expression $\mathbf{A}^{T} \mathbf{Q A}$ is a quadratic form that is positive semidefinite being equal to zero when $\bar{\alpha}_{1}=\bar{\alpha}_{2}=\cdots=\bar{\alpha}_{M}$. Hence, the comment made above with respect to the increase of the value of variance is also valid for $M>2$.

The combined fragility curve is not lognormal as explained earlier. It seems reasonable to assume, however, that the combined curve is lognormal with the mean and variance estimated, respectively, by (30) and (32). This approximation is expected to be particularly valid when the bridges under construction are designed using the same design codes. A combined fragility curve for Bridges 1 and 2 as also shown in Fig. 9 (solid curve) is in fact indistinguishable from the lognormal distribution with the median computed (in approximation) from (21) and log-standard deviation from (26).

The fragility curves developed for HEPC's bridges in Fig. 1 are based on the hypothetically homogeneous population of a sample size equal to 770 . These are referred to as composite fragility curves in this study because they can be interpreted as composites of the combined fragility curves just introduced, each being associated with a specific bridge (or column) category. The composite fragility curve can be developed from the component combined fragility curves in the same manner, as each combined curve is derived from fragility curves of its constituent nominally identical but statistically different bridges. The first two moments of the composite fragility curves can be derived from (1) the corresponding first two moments of the combined fragility curves associated with the bridge categories in the total population; and (2) the relative size of subpopulation of each bridge category to the size of the total population.

In general, the composite fragility curves are not lognormal either. Nevertheless, the lognormal assumption also is used for composite curves in Fig. 1 for analytical convenience and for the ease in which comparisons can be made with the fragility curves developed for other structural and nonstructural systems, many of which are traditionally based on the lognormal assumption. It is noted that the lognormal hypothesis deployed in constructing Fig. 1 cannot be rejected according to the result

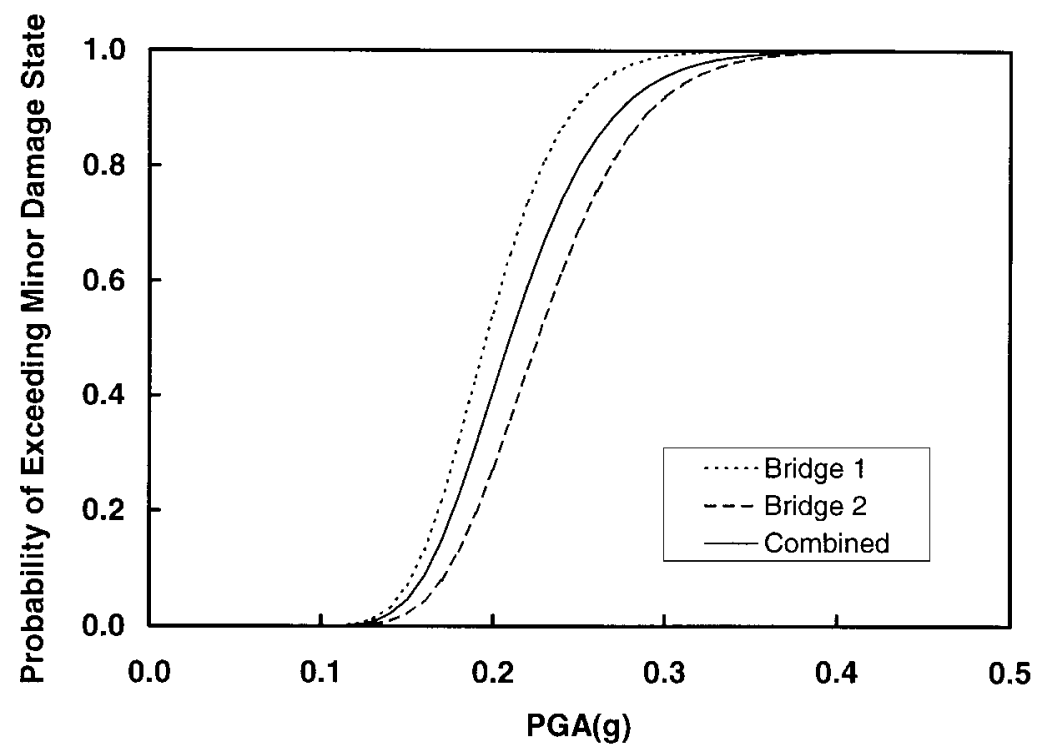

FIG. 9. Combined Fragility Curve for Bridges 1 and 2 
of the hypothesis testing involving the specific samples that were used. Whether one wishes to develop a combined or composite fragility curve, it is possible to interpret that $\mathbf{P}^{T} \mathbf{Z}$ and $\mathbf{A}^{T} \mathbf{Q A}$ in (32) are quantities that represent log-standard deviations $\beta_{U}$ and $\beta_{R}$ associated, respectively, with the uncertainty of the median value and randomness involved in the fragility curve representing each particular category of bridges (columns) with the same value of $\beta_{R}$. Here, the nuclear power plant seismic risk assessment notation and terminology are used. This interpretation is indeed compatible with that provided, for example, by Holman et al. (1987), who considered composite fragility curves for nuclear power plant equipment.

\section{CONCLUSIONS}

This study empirically developed fragility curves associated with different states of damage of HEPC's bridges under the 1995 Kobe earthquake event. Analytical fragility curves were obtained for typical bridges in the Memphis area with the aid of dynamic analysis. Two-parameter lognormal distribution functions were used to represent the fragility curves with the two parameters estimated by the maximum likelihood method. Statistical procedures were presented to test the goodness-offit hypothesis for these fragility curves and to estimate the confidence intervals of the two parameters of the lognormal distribution.

In addition, statistical interpretation of randomness and uncertainty was introduced through the notation of combined and composite fragility curves.

\section{ACKNOWLEDGMENTS}

The first writer was supported by the National Science Foundation (NSF) under Grant No. NSF-CMS-9633386, by the Federal Highway Administration under contract FHWA DTFH 1-92-C-00112 and by the Multidisciplinary Center for Earthquake Engineering Research under
Project Nos. R92629 and R92250-B. The second writer acknowledges her support by NSF Career Award CMS-9501796, and the third writer is grateful for the support by Kyunjil University, Korea.

\section{APPENDIX. REFERENCES}

Applied Technology Council (ATC). (1985). "Earthquake damage evaluation data for California." ATC-13, Redwood City, Calif.

Boore, D. M. (1983). "Stochastic simulation of high-frequency ground motions based on seismological models of the radiation spectra." Bull. Seismological Soc. of Am., 73, 1865-1894.

Holman, G. S., Chou, C. K., Shipway, G. D., and Glozman, V. (n.d.). "Compact fragility research program, Phase I. Demonstration tests." Tech. Rep. NUREG/CR-4900, Nuclear Regulatory Commission, Washington, D.C.

Hwang, H. M., and Huo, J.-R. (1996). "Simulation of earthquake acceleration time histories." Tech. Rep., Ctr. for Earthquake Res. and Information, University of Memphis, Memphis.

Idriss, I. M., and Sun, J. I. (1992). SHAKE91, a computer program for conducting equivalent linear seismic response analyses of horizontally layered soil deposits, user's manual, Ctr. for Geotech. Modeling, Dept. of Civ. and Envir. Engrg., University of California, Davis, Calif.

Jernigan, J. B., and Hwang, H. M. (1997). "Inventory and fragility analysis of Memphis bridges." Tech. Rep., Ctr. for Earthquake Res. and Information, University of Memphis, Memphis.

Nakamura, T., and Mizatani, M. (1996). "A statistical method for fragility curve development." Proc., 51st JSCE Annu. Meeting, Vol. 1-A, Japan Soc. of Civ. Engrs., Tokyo, 938-939.

Nakamura, T., Naganuma, T., Shizuma, T., and Shinozuka, M. (1998). "A study on failure probability of highway bridge by earthquake based on statistical method." Proc., 10th Japanese Earthquake Engrg. Symp., Japanese Assoc. Earthquake Engrg., Tokyo.

Shinozuka, M. (1998). "Statistical analysis of bridge fragility curves." Proc., U.S.-Italy Workshop on Protective Sys. for Bridges, Multidisciplinary Ctr. for Earthquake Engrg. Res., New York State University at Buffalo, Buffalo

Shinozuka, M., and Deodatis, G. (1991). "Simulation of stochastic processes by spectral representation.” Appl. Mech. Rev., 44(4), 191-204.

U.S. Nuclear Regulatory Commission (U.S. NRC). (1983). "PRA procedures guide." Tech. Rep. NUREG/CR-2300, Vol. 2, Washington, D.C., 11-46. 